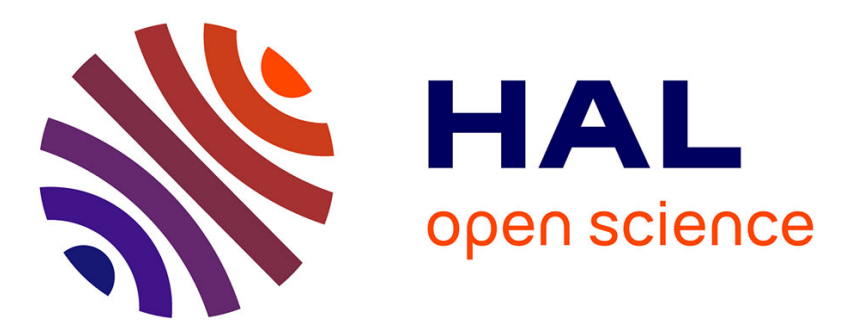

\title{
La mesure du COD des alliages d'aluminium; Difficultés rencontrées pour sa détermination
}

P. Vigier

\section{To cite this version:}

P. Vigier. La mesure du COD des alliages d'aluminium; Difficultés rencontrées pour sa détermination. Revue de Physique Appliquée, 1974, 9 (4), pp.661-665. 10.1051/rphysap:0197400904066100 . jpa00243832

\section{HAL Id: jpa-00243832 https://hal.science/jpa-00243832}

Submitted on 1 Jan 1974

HAL is a multi-disciplinary open access archive for the deposit and dissemination of scientific research documents, whether they are published or not. The documents may come from teaching and research institutions in France or abroad, or from public or private research centers.
L'archive ouverte pluridisciplinaire HAL, est destinée au dépôt et à la diffusion de documents scientifiques de niveau recherche, publiés ou non, émanant des établissements d'enseignement et de recherche français ou étrangers, des laboratoires publics ou privés. 


\title{
LA MESURE DU COD DES ALLIAGES D'ALUMINIUM ; DIFFICULTÉS RENCONTRÉES POUR SA DÉTERMINATION
}

\author{
P. VIGIER
}

\author{
Aluminium Péchiney, Centre de Recherches, 38340 Voreppe, France
}

\begin{abstract}
Résumé. - Des essais faits en vue de mesurer le COD d'alliages d'aluminium ont d'abord montré que les recommandations britanniques ne peuvent pas être suivies dans le cas de ces alliages car le centre de rotation n'a pas une position bien définie sur l'éprouvette au voisinage de l'instant critique. D'autre part, des mesures « directes » ont été faites par examen du fond de la fissure sur des coupes et par observation au microscope à balayage des surfaces de rupture ; elles ont montré que le COD est très différent suivant que l'éprouvette est encore en charge ou est déchargée, et, qu'à cause de l'hétérogénéité de la structure, le COD dépend beaucoup du plan de coupe (d'un facteur 3). Ceci fait rejeter, pour ces alliages, toute mesure faite sur coupe métallographique ou sur éprouvette déchargée ; c'est le cas par exemple des mesures faites avec l'éprouvette à deux entailles latérales adjacentes.
\end{abstract}

\begin{abstract}
Tests carried out to measure COD on aluminium alloys have first shown that the British draft is not suitable for these alloys because the centre of rotation has not a well defined position on the specimen when the COD approaches its critical value. On the other hand, "direct» measurements have been made at mid-section of the test-piece bv crack-tip examination and by SEM observation of the fractured surfaces; they have shown that COD is very different according as the specimen is still in load or is unloaded, and that, because of structural heterogeneity, COD depends much on the position of the section on which the measurements is made (about 3 times). This leads us to reject for these alloys any measurements made either on metallographic sections or on unloaded specimen ; such measurements are made for example with the two adjacent edge notches specimens.
\end{abstract}

Pour les alliages à moyenne résistance, c'est-à-dire ceux pour lesquels on ne peut pas mesurer correctement $K_{\mathrm{IC}}$, le COD est l'un des paramètres envisageables pour caractériser la résistance à la propagation des fissures.

Les problèmes concernant le COD peuvent se situer à trois niveaux différents. Tout d'abord on peut se demander si la connaissance du COD critique jointe à celle des contraintes auxquelles une structure est soumise permet d'atteindre la dimension maximale du défaut admissible dans la structure; des travaux en cours [ 1 à 5$]$ commencent à apporter des réponses à cette question. A un autre niveau, on peut se demander si le COD critique est une caractéristique intrinsèque du matériau ou bien s'il ne permet de caractériser que le produit (matériau en épaisseur donnée) ou encore s'il dépend d'autres paramètres. Enfin, à un troisième niveau, on peut se demander comment mesurer le COD critique et quelle est la signification de ce que l'on mesure.

En ce qui concerne les alliages d'aluminium à moyenne résistance, nous nous sommes posés les deux dernières questions et nous avons fait quelques essais pour tenter d'y répondre. Les essais que nous décrivons ici et qui ont pour but de chercher une méthode de mesure du COD critique applicable aux alliages d'aluminium sont de deux types :
- le projet de Norme Britannique [6] concernant la mesure du COD peut-il s'appliquer aux alliages d'aluminium ?

- le COD peut-il se mesurer directement en appliquant sa définition même, c'est-à-dire en mesurant l'ouverture de la fissure au fond de celle-ci juste avant qu'elle ne se propage.

Les éprouvettes utilisées, sollicitées en flexion, sont du type de celles que recommande l'ASTM [7] pour les mesures de $K_{\mathrm{IC}}$; en particulier, la fissure est préparée par fatigue. La section $\mathrm{B} \times \mathrm{W}$ des éprouvettes est $25 \times 50 \mathrm{~mm}$ (Fig. 1).

Les alliages essayés sont l'A-U4G1-T351, l'A-Z5GT6 et l'A-G4MC-H111. Leur composition nominale est donnée dans le tableau I. Les éprouvettes précédentes ont des dimensions suffisantes pour permettre éventuellement une mesure de $K_{\text {IC }}$ valable (au sens de l'ASTM) pour les deux premiers de ces alliages; nous espérions ainsi pouvoir vérifier expérimentalement la relation qui existe entre le COD critique et $K_{\mathrm{IC}}$.

1. Application du projet de norme britannique aux alliages d'aluminium. - Le projet de Norme Britannique [6] a été établi de sorte que le COD puisse se mesurer avec la même technique expérimentale que 


\section{TABLEAU I}

\section{Composition nominale ( $\%$ en poids) des alliages d'aluminium essayés}

\begin{tabular}{|c|c|c|c|c|c|c|c|}
\hline & $\mathrm{Cu}$ & $\mathrm{Zn}$ & $\mathrm{Mg}$ & $\mathrm{Mn}$ & $\mathrm{Cr}$ & $\mathrm{Zr}$ & Etat \\
\hline & - & - & - & - & - & - & - \\
\hline $\begin{array}{l}\text { A-U4G1-T351 } \\
\text { A-Z5G-T6 }\end{array}$ & 4,5 & 48 & 1,6 & 0,7 & ? & 015 & $\begin{array}{l}\text { Trempé, écroui, mûri } \\
\text { Trempé, revenu }\end{array}$ \\
\hline A-G4MC-H111 & & & 4,2 & 0,3 & 0,2 & & Recuit, plané \\
\hline
\end{tabular}
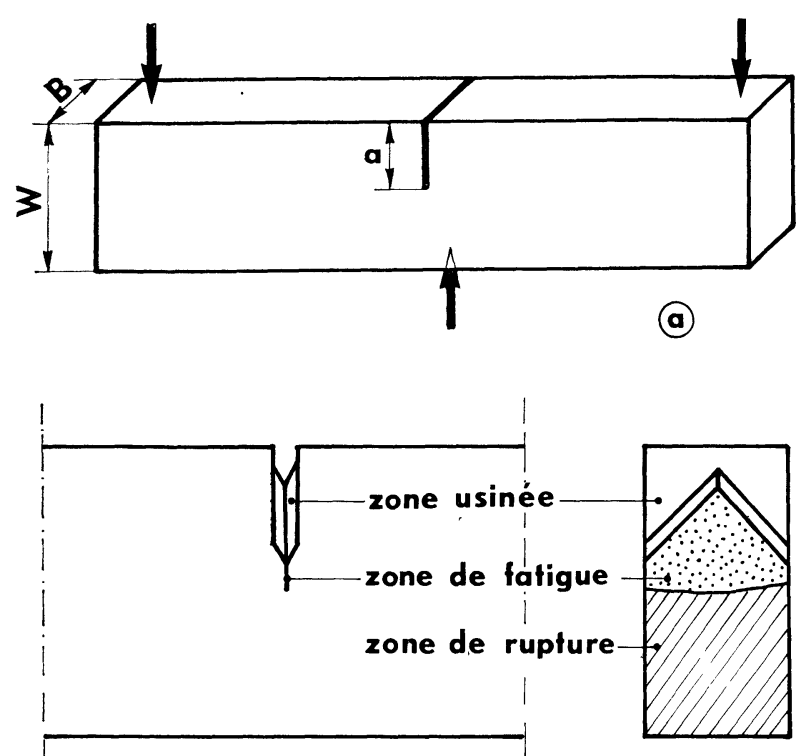

(b)

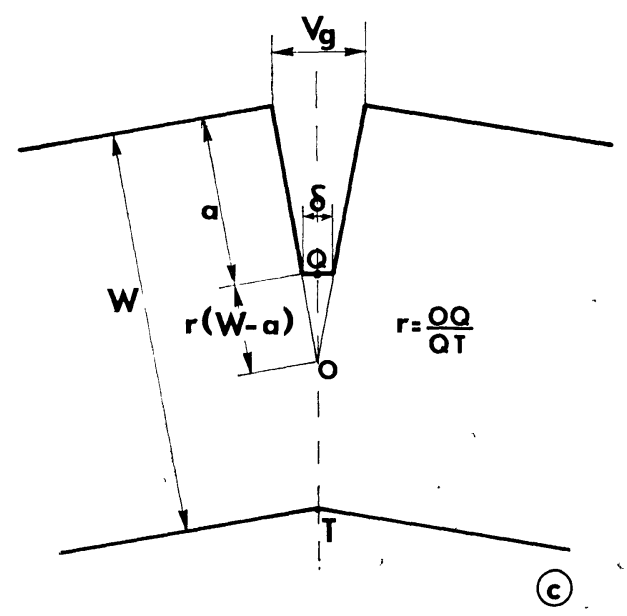

FIG. 1. - a) Eprouvette à fissure latérale unique, sollicitée en flexion, utilisée pour ces essais ; b) Détail de la fissure ; $c$ ) Schéma de la fissure lorsque l'éprouvette est fléchie.

celle que l'ASTM a normalisée pour mesurer $K_{\mathrm{IC}}$ [7] ; cette technique consiste à mesurer avec un extensomètre l'écartement $V_{g}$ des lèvres de la fissure à l'extrémité libre de celle-ci (Fig. 1c). Pour connaitre $\delta$, écartement en fond de fissure à partir de $V_{g}$, il faut connaître à chaque instant la position du centre de rotation $\mathbf{O}$ qui permet de passer de l'état actuel des lèvres de la fissure à leur état initial (lèvres accolées). Pour connaître le COD critique, $\delta_{\mathrm{C}}$, il faut en plus connaître l'instant où la propagation débute; c'est aussi un problème délicat, mais nous ne nous en préoccuperons pas dans ce chapitre. Dans le projet de Norme Britannique, il est supposé que lorsque $\delta$ a une valeur proche de $\delta_{\mathrm{C}}$, le centre de rotation $\mathrm{O}$ a une position fixe parfaitement connue et définie sur l'éprouvette et de plus indépendante du matériau. En repérant la position de ce point par le facteur de rotation $r$ défini sur la figure $1 c$ par $r=\mathrm{OQ} / \mathrm{QT}$, ceci revient à dire que $r$ a une valeur constante et bien définie lorsque $\delta$ est au voisinage de $\delta_{\mathrm{c}}$. Dans ces conditions, la mesure de $\delta$ se simplifie beaucoup puisqu'elle s'obtient à partir de la seule mesure de $V_{g}$, au lieu de nécessiter en plus dans chaque cas la connaissance de la position du centre de rotation.

Plusieurs travaux [8-9] sur des aciers ont en effet montré que $r$ était constant au voisinage de $\delta_{\mathrm{C}}$. C'est à la suite des travaux d'Ingham et coll. [8] que $r=$ Cte a été introduit dans le projet de Norme. T. Ingham a étudié comment évoluait $r$ au cours de la mise sous charge d'éprouvettes de divers types; il a trouvé que généralement $r$ avait tendance à se stabiliser vers $0,35 \sim 0,45$ avant que $\delta$ n'atteigne $\delta_{\mathrm{C}}$; le choix d'une valeur de $r$ s'est porté sur $r=\frac{1}{3}$ car la valeur de $\delta_{\mathrm{C}}$ qui en résulte (à partir de la mesure de $V_{g}$ ) est plus petite que la vraie valeur de $\delta_{\mathrm{C}}$, ce qui va dans le sens de la sécurité.

Nous avons procédé de la même manière qu'Ingham et coll. pour connaître la variation de $r$ lors de la mise sous charge de nos éprouvettes en alliage d'aluminium. Pour cela, des points de dureté servant de repères ont été disposés le long de la fissure de part et d'autre de celle-ci (Fig. 2) et des photos successives

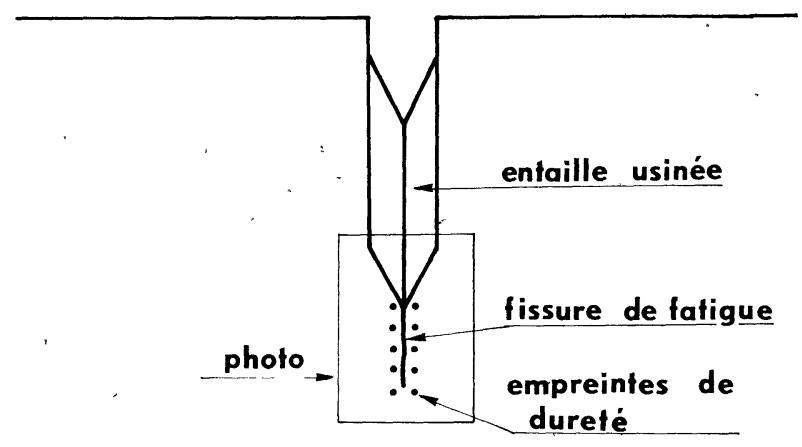

FIG. 2. - Schéma représentant la position des points de dureté de part et d'autre de la fissure pour déterminer la position du centre de rotation. 
ont été prises lors de la mise en charge. La figure 3 représente, pour l'A-U4G1, la variation de $r$ avec l'ouverture des lèvres au niveau de l'extensomètre $V_{g}$.

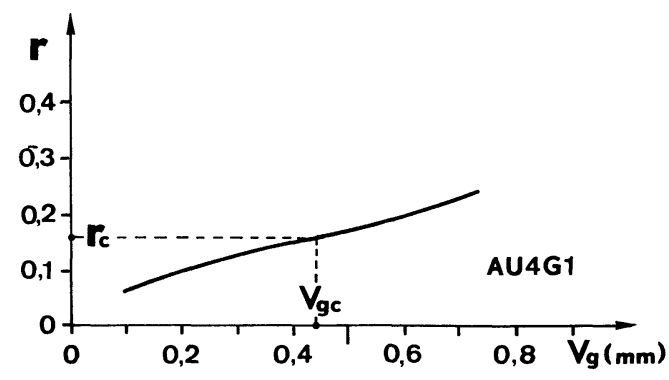

FIG. 3. - Variation du facteur de rotation $r$ en fonction de l'indication $V_{g}$ pour l'A-U4G1. Le facteur $r$ reste croissant au voisinage de l'instant critique $V_{g \mathrm{C}}$ et sa valeur est inférieure à $1 / 3$.

On constate d'abord que $r$ n'est pas stabilisé au voisinage de l'instant critique (repéré par $V_{g \mathrm{C}}$ ); $r$ augmente avec $V_{g}$ et cette variation se poursuit au-delà de $V_{g \mathrm{C}}$. D'autre part, la valeur de $r$ à l'instant critique n'est pas supérieure à $\frac{1}{3}$; la valeur de $\delta_{\mathrm{C}}$ que l'on obtiendrait à partir de $V_{g}$ et en imposant $r=\frac{1}{3}$ serait donc trop grande.

Les arguments qui ont permis de prendre $r=\frac{1}{3}$ dans le Projet Britannique ne peuvent donc pas être invoqués dans le cas des alliages d'aluminium et nous devons en conclure qu'il nous est impossible de suivre les recommandations britanniques pour mesurer le COD des alliages d'aluminium.

2. Mesure «directe» du COD par examen de l'ouverture de la fissure. - Il s'agit d'atteindre le COD critique en examinant le fond de la fissure. Si c'était à la surface de l'éprouvette que la fissure commençait à se propager, on pourrait faire cette mesure grâce à des photos prises au cours de la mise en charge de l'éprouvette; on pourrait alors facilement connaître l'instant critique et, grâce aux photos, remonter aisément à $\delta_{\mathrm{C}}$, éventuellement en procédant par interpolation ou extrapolation.

Malheureusement, la fissure commence à se propager au centre de l'éprouvette. Ce n'est donc que par examen de l'ouverture de la fissure, à cœur de l'éprouvette, que l'on pourra atteindre $\delta_{\mathrm{C}}$. Pour cela, on est conduit à prendre plusieurs éprouvettes, aussi identiques entre elles que possible, à leur faire subir des déformations à des taux différents de sorte que la fissure soit propagée sur certaines d'entre elles et non sur les autres, puis à les couper longitudinalement (Fig. 4) afin d'atteindre $\delta$ à cœur. La valeur de $\delta_{\mathrm{C}}$ s'obtient par encadrement des valeurs de $\delta$ mesurées d'une part sur les éprouvettes où il $\mathrm{y}$ a eu propagation et d'autre part sur celles où la fissure ne s'est pas encore propagée.

Pratiquement nous avons constaté que ce mode opératoire ne donnait que des valeurs très imprécises. En effet, la mesure de $\delta$ sur coupe, au microscope

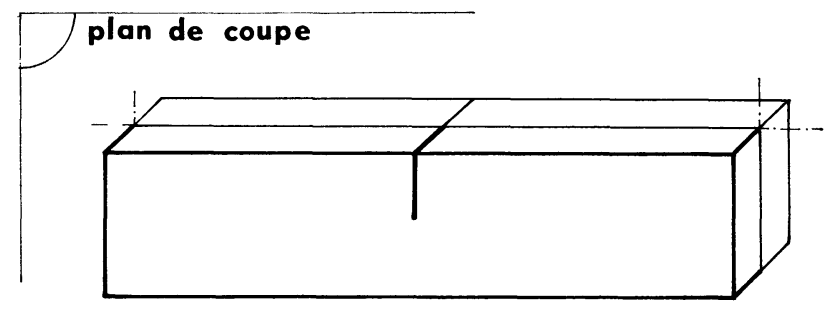

FIG. 4. - Manière dont sont coupées les éprouvettes pour examiner, au microscope optique, l'ouverture de la fissure à cœur.

optique, n'est possible que si la surface a été préalablement polie ; or, le COD critique des alliages que nous voulons mesurer est très faible, de l'ordre de 30 à $120 \mu$ suivant les alliages, comme nous l'indiquons plus loin; dans ces conditions, le polissage perturbe trop le fond de la fissure pour espérer une bonne précision de cette mesure directe.

Nous avons pensé obtenir une meilleure valeur de $\delta_{\mathrm{C}}$ toujours en procédant par encadrement à partir des mêmes éprouvettes coupées longitudinalement, mais en modifiant sur chacune d'elles la manière de mesurer $\delta$. Pour une éprouvette donnée, par exemple une éprouvette où la fissure ne s'est pas encore propagée, on pensait obtenir $\delta$ en extrapolant jusqu'au fond de la fissure l'écartement $V(z)$ de ses lèvres, cet écartement étant pris entre des cotes qui correspondent respectivement, comme le montre la figure 5 , au bord de l'éprouvette et à un point situé au voisinage du fond.

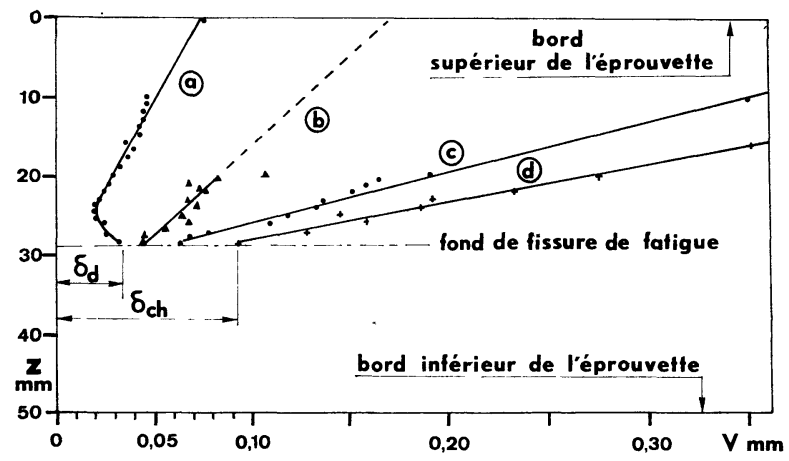

Fig. 5. - Ecartement des lèvres de la fissure depuis le voisinage du fond de la fissure jusqu'au bord de l'éprouvette : Courbe a) mesure faite sur coupe à cœur d'une éprouvette préalablement sollicitée en flexion puis déchargée ; le COD mesuré est alors $\delta_{\mathrm{d}}$; Courbes $b$ ) et c) modification de cet écartement lorsque l'éprouvette coupée longitudinalement est à nouveau mise en fléxion; Courbe d) correspond à un état de flexion identique à celui qu'avait subi l'éprouvette lors de sa première mise sous charge ; le COD mesuré est alors $\delta_{\mathrm{ch}}$.

L'examen des. lèvres de la fissure au voisinage de son fond nous a malheureusement convaincu que cette extrapolation était impossible. Si l'on veut néanmoins la faire, on trouve dans certains cas un COD négatif. En effet, au voisinage du fond de la fissure, les lèvres sont incurvées comme le montre la courbe a de la figure 5 . 
Cette courbure est sans doute due à la nature particulière de la zone plastique au fond de la fissure ; cette zone doit être analogue à celle qui existe en avant d'une fissure de fatigue. En effet, notre éprouvette a subi d'abord une mise sous charge, puis elle a été déchargée avant d'être coupée longitudinalement et observée. L'existence de la zone plastique "cyclique» déformée en compression, intérieure à la zone plastique "monotone" décrite par exemple par $\mathrm{H}$. de Leiris, R. M. Pelloux et C. Bathias [10-11] pourrait expliquer la forme des lèvres.

Dans cette hypothèse, le profil des lèvres devrait redevenir rectiligne si l'éprouvette est mise à nouveau en charge. Pour vérifier cela, nous avons observé sur l'une des moitiés d'une éprouvette coupée longitudinalement - éprouvette où la fissure ne s'était pas encore propagée - comment évoluait le profil des lèvres de la fissure lorsque cette demi-éprouvette était remise en charge.

La figure 5 montre divers profils correspondant à divers états de flexion.

On constate qu'effectivement, comme nous l'avions supposé, le profil redevient rectiligne lorsque l'éprouvette est remise en charge (courbes $b, c, d$ ). Mais on constate aussi que l'ouverture en fond de fissure augmente avec le chargement; cela signifie que la valeur de $\delta$ obtenue par une mesure sur éprouvette déchargée n'est pas la valeur que l'on souhaiterait obtenir, c'est-à-dire celle qui existait à cœur de l'éprouvette entière et sous charge.

On peut espérer connaître la valeur de $\delta$ qui existait à cœur de l'éprouvette lorsqu'elle était entière et non encore déchargée en chargeant la demi-éprouvette de sorte que sa déformation globale - en flexion soit la même que celle qu'avait l'éprouvette entière lors de la première mise en charge. La courbe $\mathrm{d}$ sur la figure 5 correspond à cet état de flexion. On remarque que la valeur de $\delta$ alors obtenue est de 2 à 3 fois plus élevée que celle en décharge; cette différence est trop importante pour pouvoir être simplement attribuée à une déformation élastique du fond de l'entaille.

Donc, malgré le manque de précision de nos mesures et malgré le fait que certain point soit discutable (ouverture des lèvres sur la demi-éprouvette rechargée identique à celle sur l'éprouvette entière avant décharge), on peut conclure que $\delta$ en charge est très différent (d'un facteur supérieur à 2) de $\delta$ en décharge.

Cette première conclusion nous conduit à la conclusion suivante: il n'est pas possible d'utiliser les éprouvettes à deux entailles adjacentes pour mesurer le COD des alliages d'aluminium que nous avons étudiés. Rappelons en effet que cette éprouvette à double entaille est conçue dans le but de mesurer le COD sur celle des deux entailles qui ne s'est pas propagée, cette mesure s'effectuant sur coupe métallographique, au cœur de l'éprouvette déchargée.
3. Mesure du COD, après rupture totale, par examen des surfaces de rupture. - Certains auteurs, dont A. S. Tetelman [12], admettent que lorsque la fissure a commencé à se propager, il n'y a plus aucune déformation du métal à l'endroit où la fissure était avant de commencer à se propager. Donc, en se référant à la figure 6 , qui schématise l'évolution du fond de la fissure au cours de la mise en charge, la valeur du COD critique, représentée par $\mathrm{AB}$ sur le schéma $\mathrm{c}$, est aussi égale à la somme $\mathrm{AC}+\mathrm{DB}$ sur les schémas e et $\mathrm{f}$.

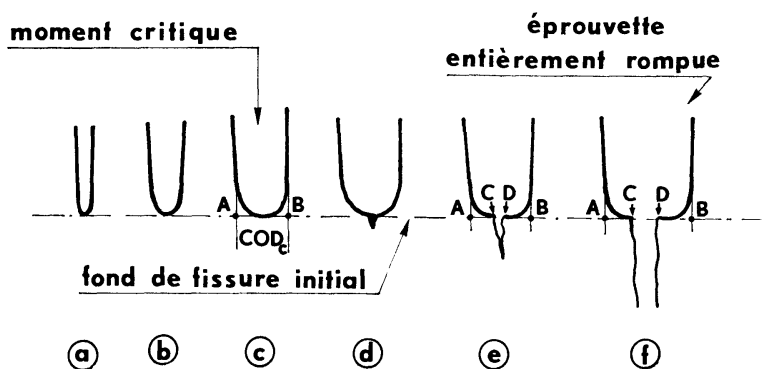

FIG. 6. - Schéma montrant des stades successifs du fond de la fissure lorsque l'éprouvette est mise en charge.

Lorsque l'éprouvette est totalement rompue par propagation complète de la fissure, ces marches AC et DB se retrouvent sur chacune des surfaces de rupture. Le COD peut donc se déterminer, "comme le montre la figure 7 , en mesurant la hauteur de ces marches sur les deux surfaces de rupture. L'une des principales difficultés consiste à retrouver sur les deux surfaces les points qui se correspondent, c'est-à-dire ceux qui se trouvaient en contact avant rupture.

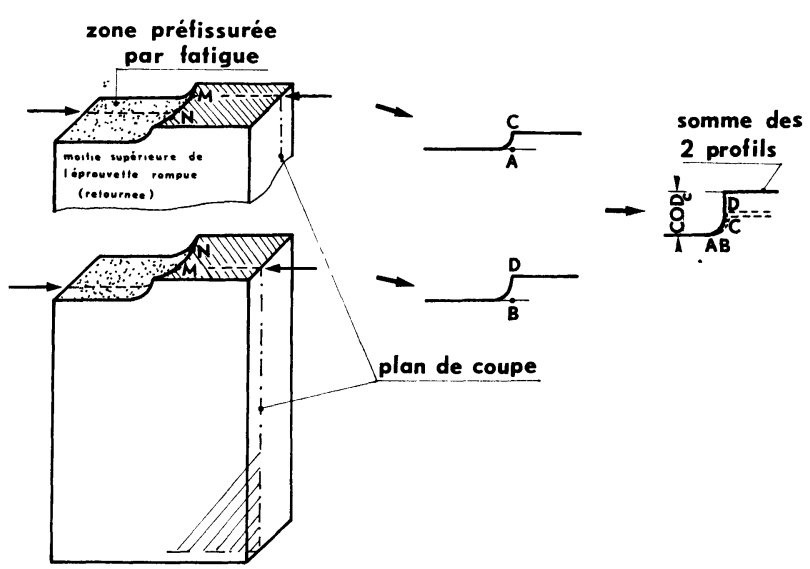

FIG. 7. - Principe de mesure du COD critique par examen des deux surfaces de rupture.

L'avantage essentiel de cette méthode est qu'elle donne directement la valeur critique du COD, alors que les méthodes précédentes nécessitaient, pour atteindre $\delta_{\mathrm{C}}$, de connaître à la fois $\delta$ et l'instant critique (dans le cas de la méthode extensométrique) ou de procéder par encadrement. 
Nos essais ont consisté à mesurer la hauteur des marches qui se correspondent en examinant les surfaces de rupture au microscope à balayage et en procédant par stéréographie sur des photographies prises à un grandissement de l'ordre de 200.

Nous avons constaté que lorsqu'on se déplace le long de la marche $\mathrm{MN}$ d'une même éprouvette (Fig. 7), tout en restant dans la zone centrale (zone en déformation plane), le COD critique varie beaucoup ; cette variation est bien supérieure aux incertitudes de nos mesures puisque l'on peut trouver un facteur 3 entre les valeurs extrêmes. Cette variation se relie à la microstructure du métal qui, effectivement, est hétérogène à l'échelle du COD.

Ce résultat montre que toute mesure «locale» faite sur coupe métallographique conduit nécessairement dans le cas des alliages que nous avons étudiés, à une valeur très imprécise du COD, à un facteur 3 près d'après nos essais.

Donc, pour mesurer le COD des alliages d'aluminium, il faut éliminer les techniques qui font intervenir des examens sur coupe métallographique. En particulier, nous ne pourrons pas utiliser l'éprouvette à double entaille; nous avions déjà donné une première raison pour rejeter ce type d'essai. De même, il n'est pas possible d'obtenir une mesure correcte en développant la technique que nous avons décrite au chapitre précédent, c'est pourquoi nous n'avions pas donné les valeurs du COD mesurées ainsi.

Néanmoins, en tenant compte de l'ensemble de nos essais, on peut indiquer un ordre de grandeur du COD des alliages sur lesquels nous avons travaillé ; le COD est voisin de 120 microns pour l'A-G4MCH111 et de l'ordre de 30 à 80 microns pour l'A-Z5G-T6 et l'A-U4G1-T351. Il convient de considérer ces valeurs avec circonspection.

4. Conclusion. - Ces quelques essais qui avaient pour but de préciser comment appliquer et mesurer le COD critique d'alliages d'aluminium permettent de tirer quelques conclusions, qu'il convient bien sûr de ne pas généraliser à d'autres alliages :

- le projet de Norme Britannique ne peut pas être utilisé ;
- l'ouverture du fond d'une fissure est très différente suivant que l'éprouvette est en charge ou déchargée. Ceci nous conduit à rejeter l'éprouvette à deux entailles adjacentes pour mesurer le COD critique de ces alliages;

- le COD critique mesuré localement varie dans de très grandes proportions en fonction de la microstructure locale, ce qui, dans notre cas, conduit à rejeter toute mesure sur coupe métallographique comme étant trop imprécise.

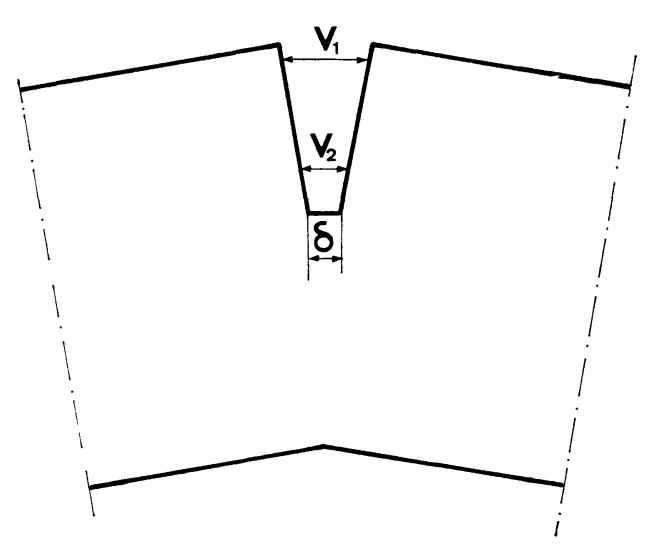

FIG. 8. - Mesure du COD avec deux extensomètres qui donnent $V_{1}$ et $V_{2}$.

Nous pensons que la mesure du COD à l'aide de deux extensomètres disposés comme l'indique la figure 8 pourrait donner une valeur correcte; le COD se déduit de la connaissance de $V_{1}$, de $V_{2}$ et de l'instant critique (ce qui est difficile à connaître) ; nous allons poursuivre nos essais dans ce sens.

Remerciements. - Les essais ont été effectués par R. Brault et J. M. Dujardin au cours d'un stage de longue durée faisant partie de leur $3^{\mathrm{e}}$ année d'Ecole d'Ingénieur. Je remercie également J. M. Gjurasevic qui a grandement contribué aux mesures faites au microscope à balayage, ainsi que A. Arcis qui a participé à l'ensemble des essais.

\section{Bibliographie}

[1] Wells, A. A., Br. Welding Journal 10 (1963) 563-570.

[2] Burdekin, F. M., Stone, D. E. W., J. of Strain Analysis 1 (1966) $145-153$

[3] Burdekin, F. M., Dawes, M. G., Practical application of fracture mechanics to pressure vessel technology. Paper C5/71, Londres, mai 1971.

[4] Frederick, G., Revue de la Soudure (Belge) 3 (1971) 187-197.

[5] Pluvinage, G., Thèse, Lille, 4 mai 1973.

[6] British Standards Institution; Draft for development 19 (1972) Methods for Crack Opening Displacement (COD) Testing.

[7] ASTM, Tentative method of test for plane strain fracture toughness of metallic materials, E399-70 T.
[8] Ingham, T., Egan, G. R., Elliot, D., Harrison, T. C., Practical application of fracture mechanics to pressure vessel technology, Paper C54/71, Londres, mai 1971.

[9] Guittet, J. C., Sifferlen, R., Communication aux Journées Métallurgiques d'Automne, Paris, octobre 1973.

[10] Bathias, C., Pelloux, R. M., 15e Colloque de Métallurgie, Saclay, 1972.

[11] De Leiris, H., Bathias, C., Conférence prononcée à Mons, juin 1973.

[12] Robinson, J. N., Tetelman, A. S., Third International Conf. on Fracture, Münich, avril 1973, Paper II-421. 